ISSN 1980-5098

\title{
IMPACTOS DA INVASÃO POR Cryptostegia madagascariensis Bojer ex Decne. (Apocynaceae JusS.) EM REMANESCENTES DE CAATINGA NO MUNICÍPIO DE IBARETAMA, CEARÁ, BRASIL
}

IMPACTS OF THE INVASION BY Cryptostegia madagascariensis Bojer ex Decne. (Apocynaceae Juss.) IN THE REMNANT OF CAATINGA IN THE TOWN OF IBARETAMA, CEARÁ STATE, BRASIL

\author{
Flaubert Queiroga de Sousa ${ }^{1}$ Leonaldo Alves de Andrade ${ }^{2}$ Klerton Rodrigues Forte Xavier ${ }^{3}$ \\ Patrícia Cândido da Cruz Silva ${ }^{4}$ Manoel Bandeira de Albuquerque ${ }^{5}$
}

\section{RESUMO}

Cryptostegia madagascariensis Bojer ex Decne. é uma espécie de porte arbustivo capaz de formar maciços populacionais em diversos nichos da caatinga e ecossistemas associados, sobretudo nos sítios mais úmidos, podendo alterar as características da vegetação nativa, causando o desaparecimento das espécies autóctones. Este estudo teve como objetivo identificar os impactos causados pela invasora sobre a composição florística e a estrutura do componente arbustivo-arbóreo. Foram selecionadas três áreas de investigação, sendo denominadas de Ambiente I - área com forte intensidade de invasão, Ambiente II - área de transição entre o I e o III (média intensidade) e o Ambiente III - área com baixa intensidade de invasão. Para o inventário dessas áreas foi empregado o método de amostragem por pontos (Método dos Quadrantes), sendo considerados adultos os indivíduos com diâmetro ao nível do solo (DNS) maior ou igual a $3 \mathrm{~cm}$ e altura maior ou igual a $1 \mathrm{~m}$. Para a avaliação da estrutura foram analisados os parâmetros fitossociológicos convencionais, além dos índices de Diversidade, de Equabilidade e de Impacto Ambiental de Exóticas (IIAE). No Ambiente I, Cryptostegia madagascariensis foi responsável por $82 \%$ da densidade, enquanto que nos Ambientes II e III, a densidade da espécie foi bastante reduzida (14,2\% e 9,6\%, respectivamente). A diversidade, pelo Índice de Shannon-Weiner, foi baixa para o Ambiente I $(0,85)$ e maior para os Ambientes II e III (2,67 e 2,50, respectivamente). Os resultados demostram que Cryptostegia madagascariensis afeta drasticamente a diversidade e a estrutura das comunidades invadidas, ocasionando perda da biodiversidade autóctone da caatinga.

Palavras-chave: espécie exótica; invasão biológica; semiárido.

\begin{abstract}
Cryptostegia madagascariensis Bojer ex Decne. is a shrubby species capable of forming populational massifs in several niches of caatinga and its related ecosystems, especially in the most humid sites, that may change the characteristics of native vegetation, triggering the disappearance of autochthonous species. This study aimed to identify the impacts caused by the invader on the floristic composition and the structure of the shrub-arboreal component. Three areas of investigation were selected, they were termed by: Ambience

1 Engenheiro Agrônomo, Dr., Departamento de Fitotecnia e Ciências Ambientais, Universidade Federal da Paraíba, Campus II, Caixa Postal 66, CEP 58397-000, Areia (PB), Brasil. flaubertqueiroga@yahoo.com.br

2 Engenheiro Agrônomo, Dr., Professor Associado do Departamento de Fitotecnia e Ciências Ambientais, Universidade Federal da Paraíba, Campus II, Caixa Postal 66, CEP 58397-000, Areia (PB), Brasil. landrade@cca.ufpb.br

3 Biólogo, Dr., Departamento de Fitotecnia e Ciências Ambientais, Universidade Federal da Paraíba, Campus II, Caixa Postal 66, CEP 58397-000, Areia (PB), Brasil. klertonxavier@hotmail.com

4 Engenheira Agrônoma, Me., Departamento de Fitotecnia e Ciências Ambientais, Universidade Federal da Paraíba, Campus II, Caixa Postal 66, CEP 58397-000, Areia (PB), Brasil. patriciachamaviva@hotmail.com

5 Engenheiro Agrônomo, Dr., Professor Adjunto do Departamento de Fitotecnia e Ciências Ambientais, Universidade Federal da Paraíba, Campus II, Caixa Postal 66, CEP 58397-000, Areia (PB), Brasil. mbalbuquerque@yahoo.com.br
\end{abstract}

Recebido para publicação em 20/11/2014 e aceito em 30/05/2016 
I - area with a high intensity of invasion, Ambience II - the transition area between I and III (medium intensity), and Ambience III - area with a low intensity of invasion. For the inventory of those areas, it was used the point-centered sampling method (Quarter Method), considering adults those plants that presented the diameter at ground level (DGL) greater than or equal to $3 \mathrm{~cm}$, and height greater than or equal to $1 \mathrm{~m}$. For the evaluation of the structure the conventional phytosociological parameters were analyzed, beyond indices of Diversity, Equitability and Environmental Impact of Exotics (IEIE). In the Ambience, I Cryptostegia madagascariensis was responsible for $82 \%$ of density, while in the Ambiences II and III, the density of the species was severally reduced (14.2\% and 9.6\%, respectively). The diversity, according to the Shannon-Weiner index, was low for the Ambience I (0.85) and higher for the Ambiences II and III (2.67 e 2.50, respectively). Results demonstrate that Cryptostegia madagascariensis affects severely the diversity and the structure of invaded communities, causing loss in the autochthonal biodiversity of caatinga.

Keywords: exotic species; biological invasion; semiarid.

\section{INTRODUÇÃO}

As espécies exóticas quando inseridas em novo habitat podem encontrar condições ambientais adequadas à sua sobrevivência, tornando-se mais eficientes que as espécies nativas quanto ao uso dos recursos do meio (LOPES, 2009; OLIVEIRA; SILVA JÚNIOR; RIBEIRO, 2011), podendo causar sérias consequências para a ecologia, economia, como também para a saúde da sociedade (OLIVEIRA; PEREIRA, 2010). Devido a esses fatores negativos o tema vem chamando atenção de cientistas e autoridades em todo o mundo. No Brasil, os problemas de invasão biológica por espécies exóticas já são constatados em todos os biomas, em diferentes intensidades. De acordo com Zenni e Ziller (2011), atualmente há mais de cem espécies de plantas exóticas reconhecidas como invasoras e um número muito superior de plantas introduzidas espalhadas pelos diferentes biomas brasileiros. Na região Nordeste do Brasil, várias espécies exóticas já foram identificadas como invasoras e os seus impactos avaliados [Prosopis juliflora (Sw.) DC.; Sesbania virgata L.; Sporobolus indicus L.; Parkinsonia aculeata L.], sendo constatados vários danos sobre a vegetação em que elas se estabelecem, principalmente, sobre a fitodiversidade autóctone da caatinga (ANDRADE, 2013).

$\mathrm{Na}$ vegetação de caatinga, mais particularmente a que abrange o estado do Ceará, a espécie exótica Cryptostegia madagascariensis Bojer ex Decne., pertencente à família Apocynaceae (ENDRESS; BRUYNS, 2000) é popularmente conhecida na região como unha-do-cão e vem apresentando grande potencial invasor, sobretudo devido à fácil dispersão das sementes promovida pelo vento aliado a seu elevado índice de germinação (VIEIRA et al., 2004). Esta espécie é uma planta lenhosa, perene, policaulescente e de porte arbustivo com cerca de 2,0 a 3,0 m de altura (KLACKENBERG, 2001; KRITICOS et al., 2003). Apesar de arbustiva, essa espécie desenvolve ramos trepadores, que, se não podados, podem alcançar alturas consideráveis, usando como suporte, por exemplo, outras plantas (VIEIRA et al., 2004; ANDRADE, 2013). Com esse hábito de crescimento, essa espécie vem causando sérios danos à vegetação das matas ciliares da caatinga cearense, competindo principalmente com a carnaubeira [Copernicia prunifera (Mill.) H. E. Moore], matando-a por sombreamento já que a mesma desenvolve ramos trepadores, com grande produção de biomassa, que impedem a passagem de luz, causa asfixia, estrangulamento e tombamento, provocando a morte dos espécimes atingidos (ANDRADE, 2013); ocasionando prejuízos econômicos para os produtores rurais, já que grande parte vive da extração dos produtos extraídos da carnaubeira (SILVA et al., 2012).

Portanto, em meio a essa problemática, é de fundamental importância estabelecer investigações e estratégias que possam diminuir os impactos causados pela espécie invasora no âmbito socioeconômicoambiental. O levantamento florístico-fitossociológico pode auxiliar no monitoramento das eventuais alterações na estrutura da vegetação nativa causada pela Cryptostegia madagascariensis, pois é possível evidenciar a composição e a estrutura em ambientes com e sem invasão proporcionando identificar níveis de impacto e nortear estratégias para manejo dos remanescentes atingidos (MARANGON et al., 2007; XAVIER, 2009).

Dessa forma, o presente estudo objetivou identificar os impactos ocasionados pela invasora sobre a fitodiversidade e a estrutura do componente arbustivo-arbóreo em remanescentes de caatinga, no município de Ibaretama, no Estado do Ceará. 


\section{MATERIAL E MÉTODOS}

\section{Caracterização do local}

O estudo foi desenvolvido em uma Área de Preservação Permanente (APP) no estado do Ceará, localizada no município de Ibaretama - Fazenda Triunfo, com coordenadas geográficas $4^{\circ} 44^{\prime} 10^{\prime \prime} \mathrm{S}$ e 38 45'02" W e altitude média de 109 m. O clima da região, segundo classificação de Köppen, é o BShw' (tropical quente semiárido), com temperatura média entre $26^{\circ}$ e $28^{\circ} \mathrm{C}$ e pluviosidade média de 838,1 $\mathrm{mm}$, tendo como período chuvoso os meses de janeiro a abril. O relevo da região é suave ondulado, os solos predominantes são os Litólicos, Planossolo Solódico e Podzólico Vermelho-Amarelo e a vegetação predominante é composta por Caatinga Arbustiva Densa e Floresta Caducifólia Espinhosa (FUNDAÇÃO CEARENSE DE METEOROLOGIA E RECURSOS HÍDRICOS; INSTITUTO DE PESQUISA E ESTRATÉGIA ECONÔMICA DO CEARÁ, 2013).

Para avaliar os impactos causados por Cryptostegia madagascariensis sobre a vegetação arbustivoarbórea autóctone da caatinga, foram selecionadas três áreas, denominadas Ambientes (Figura 1):

- Ambiente I (área com forte intensidade de invasão): área com aproximadamente 20 ha inventariados com forte presença da invasora Cryptostegia madagascariensis. Segundo informações do proprietário da fazenda, a área sofreu o último corte raso em toda sua extensão há mais de quatro anos, sendo que, a partir daí, observou-se um rápido estabelecimento da invasora;

- Ambiente II (área de transição entre os Ambientes I e III): área com aproximadamente 15 ha inventariados, abrange a margem direita da vegetação ciliar do rio que corta a fazenda. Este ambiente é caracterizado por apresentar indivíduos da invasora entremeados à vegetação nativa, mas com menos intensidade do que o Ambiente I. Segundo o proprietário, essa área sofreu cortes esporádicos e também foi usada para pecuária extensiva, sendo que a maior parte encontra-se conservada há mais de trinta anos;

- Ambiente III (área com baixa intensidade de invasão): área de caatinga relativamente bem conservada há mais de quarenta anos, com aproximadamente 10 ha inventariados, abrange a margem esquerda da vegetação ciliar do rio. Há também a presença da espécie invasora, porém, em menor intensidade em

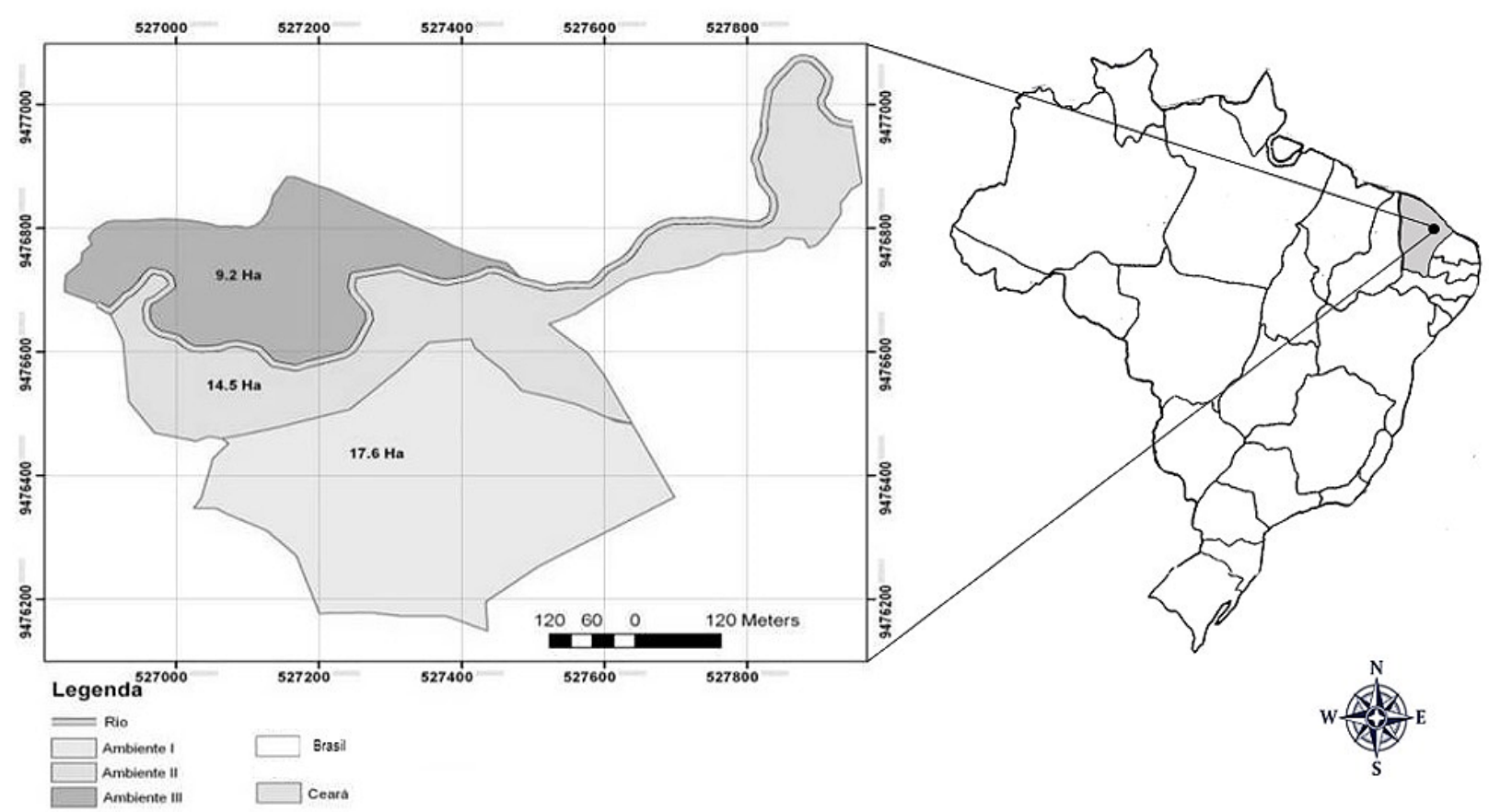

FIGURA 1: Áreas de estudo localizadas na fazenda Triunfo, município de Ibaretama, Ceará, região nordeste do Brasil.

FIGURE 1: Study area located in farm of Triunfo, town of Ibaretama, state of Ceará, northeastern Brazil. 
relação aos demais ambientes. A área é caracterizada por não sofrer cortes para extração de lenha e também por não ser observada a prática do pastejo.

\section{Procedimentos}

No levantamento florístico-estrutural foi utilizado o método de amostragem por pontos - Método dos Quadrantes (COTTAM; CURTIS, 1956; MULLER-DOMBOIS; ELLEMBERG, 1974). As unidades amostrais foram plotadas aleatoriamente, sendo 130 pontos amostrais por Ambiente. Em cada quadrante alocado foram amostrados quatro indivíduos arbustivos e/ou arbóreos mais próximos ao ponto. No presente trabalho, os indivíduos foram considerados adultos quando possuíam diâmetro, em nível do solo (DNS), maior ou igual a $3 \mathrm{~cm}$ e altura maior ou igual a $1 \mathrm{~m}$ (RODAL; SAMPAIO; FIGUEIREDO, 2013). O diâmetro dos indivíduos inventariados foi tomado utilizando-se uma suta dendrométrica e a altura estimada em metros (no nível do solo ao ápice da copa).

A estrutura da comunidade arbustivo-arbórea foi calculada por meio dos parâmetros fitossociológicos convencionais: a área basal, os valores absolutos e relativos de densidade, frequência e dominância; o valor de cobertura (VC) e o valor de importância (VI) (MULLER-DUMBOIS; ELLEMBERG, 1974, MATEUCCI; COLMA, 1982).

A diversidade e a equabilidade foram calculadas pelos Índices de Shannon-Wiener $\left(H^{\prime}\right)$ (SHANNON; WEAVER, 1949) e de Pielou (ODUM, 1988), respectivamente. O impacto da espécie exótica sobre a comunidade estudada foi analisado por meio do índice de impacto ambiental de exóticas, efetuado a partir do cálculo do coeficiente do impacto ambiental (REASER et al., 2007).

A lista florística foi organizada de acordo com o Sistema APG III (ANGIOSPERM PHYLOGENY GROUP III, 2009) para famílias, disponível na base de dados Tropicos $^{\circledR}$ (2012) do Missouri Botanical Garden, Saint Louis, Missouri, USA e a lista dos autores das espécies segundo Brummitt e Powell (1992). As análises estruturais foram realizadas utilizando-se do Software Mata Nativa $2^{\circ}$ (PARQUE DE CIÊNCIA E TECNOLOGIA, 2002) e o mapa das áreas obtido através do programa ArcZIS10.

\section{RESULTADOS E DISCUSSÃO}

No total, foram inventariados 1.560 indivíduos pertencentes a 14 famílias, 29 gêneros e 36 espécies, dos quais 28 foram identificados em nível de espécie, seis até o nível de gênero, dois até o nível família e duas ficaram indeterminados (Tabela 1).

Observando a distribuição taxonômica relativa aos três ambientes, nota-se uma diminuição drástica no número de famílias, gêneros e espécies do Ambiente I para os demais Ambientes. Enquanto o Ambiente I representa $41 \%$ das famílias amostradas em todo o estudo, Ambiente II e III representam $77 \%$ e $86 \%$, respectivamente. Em relação aos gêneros, a redução foi ainda maior, dos 29 gêneros catalogados, 35\% foram observados no Ambiente I, 79\% no Ambiente II e 82\% no Ambiente III. Em se tratando de espécie, o comportamento seguiu a mesma tendência, tendo ocorrido no Ambiente I apenas 30\% do total de espécies inventariadas, enquanto no Ambiente II e III ocorreram, em cada, $72 \%$ das espécies amostradas.

Mesmo sendo áreas adjacentes, a forte presença da espécie exótica invasora Cryptostegia madagascariensis no Ambiente I afetou negativamente a fitodiversidade autóctone, o que fica evidenciado quando comparadas as riquezas nos Ambientes estudados. A redução da riqueza de espécies, devido ao processo de invasão biológica em áreas de Caatinga, também foi constatada por Pegado et al. (2006), Andrade, Fabricante e Oliveira (2009; 2010), Souza et al. (2011) e Andrade (2013).

A comunidade vegetal amostrada no Ambiente I apresentou uma distância média indivíduoponto de 3,12 m, correspondendo a uma densidade total de 1.889 ind.ha $^{-1}$. Já a comunidade amostrada no Ambiente II apresentou uma distância média indivíduo-ponto de $1,09 \mathrm{~m}$, correspondendo a uma densidade total de 10.651 ind.ha ${ }^{-1}$. Enquanto que no Ambiente III, a distância média indivíduo-ponto foi de 2,08 m, correspondendo a uma densidade total de 3.579 ind.ha $^{-1}$ (Tabela 2).

O Ambiente I proporcionou uma distância média entre o ponto central do quadrante e o indivíduo mais próximo maior que os dos demais Ambientes (II e III), indicando que o grau de perturbação, bem como os danos originados pela invasora causaram danos à comunidade vegetal autóctone. Segundo Moro 
TABELA 1: Famílias e espécies botânicas amostradas nos ambientes estudados, município de Ibaretama, Ceará, Brasil.

TABLE 1: Families and botanical species sampled in the three studied sites, town of Ibaretama, state of Ceará state, Brazil.

\begin{tabular}{|c|c|c|c|c|}
\hline \multirow{2}{*}{ Família/Espécie } & \multirow{2}{*}{ Nome vulgar } & \multicolumn{3}{|c|}{ Ambientes } \\
\hline & & I & II & III \\
\hline \multicolumn{5}{|l|}{ ANACARDIACEAE } \\
\hline Myracrodruon urundeuva Allemão & Aroeira & & $\mathrm{X}$ & $\mathrm{X}$ \\
\hline Spondias mombin L. & Cajazeira & & & $\mathrm{X}$ \\
\hline \multicolumn{5}{|l|}{ APOCYNACEAE } \\
\hline Aspidosperma pyrifolium Mart. & Pereiro & $\mathrm{X}$ & $\mathrm{X}$ & $\mathrm{X}$ \\
\hline Cryptostegia madagascariensis Bojer ex Decne & Unha-do-cão & $\mathrm{X}$ & $\mathrm{X}$ & $\mathrm{X}$ \\
\hline \multicolumn{5}{|l|}{ ARECACEAE } \\
\hline Copernicia prunifera (Mill.) H. E. Moore & Carnaúba & $\mathrm{X}$ & $\mathrm{X}$ & $\mathrm{X}$ \\
\hline \multicolumn{5}{|l|}{ BORAGINACEAE } \\
\hline Auxemma oncocalyx (Allemao) Baill. & Pau-branco & $\mathrm{X}$ & $\mathrm{X}$ & $\mathrm{X}$ \\
\hline \multicolumn{5}{|l|}{ BURSERACEAE } \\
\hline Commiphora leptophloeos (Mart.) J.B. Gillet & Imburana & & & $\mathrm{X}$ \\
\hline \multicolumn{5}{|l|}{ CACTACEAE } \\
\hline Cereus jamacaru DC. & Mandacaru & $\mathrm{X}$ & $\mathrm{X}$ & $\mathrm{X}$ \\
\hline \multicolumn{5}{|l|}{ CAPPARACEAE } \\
\hline Crateva tapia var. glauca & Trapiá & & $\mathrm{X}$ & \\
\hline \multicolumn{5}{|l|}{ COMBRETACEAE } \\
\hline Combretum leprosum Mart. & Mufumbo & $\mathrm{X}$ & $\mathrm{X}$ & $\mathrm{X}$ \\
\hline \multicolumn{5}{|l|}{ EUPHORBIACEAE } \\
\hline Croton blanchetianus Baill. & Marmeleiro & $\mathrm{X}$ & $\mathrm{X}$ & $\mathrm{X}$ \\
\hline Croton campestris A. St. Hil. & Velame & $\mathrm{X}$ & $\mathrm{X}$ & $\mathrm{X}$ \\
\hline Croton sp. & Quebra-faca & & & $\mathrm{X}$ \\
\hline Jatropha mollissima (Pohl) Baill. & Pinhão-bravo & $\mathrm{X}$ & $\mathrm{X}$ & $\mathrm{X}$ \\
\hline Indeterminado 2 & Açoita-cavalo & & $\mathrm{X}$ & \\
\hline \multicolumn{5}{|l|}{ FABACEAE - CAESALPINIOIDEAE } \\
\hline Bauhinia cheilantha (Bong.) Steud. & Mororó & & $\mathrm{X}$ & $\mathrm{X}$ \\
\hline Libidibia ferrea (Mart.) L.P. Queiroz & Pau-ferro & & $\mathrm{X}$ & \\
\hline Parkinsonia aculeata $\mathrm{L}$. & Turco & $\mathrm{X}$ & & \\
\hline Poincianella pyramidalis (Tul.) L. P. Queiroz & Catingueira & $\mathrm{X}$ & $\mathrm{X}$ & $\mathrm{X}$ \\
\hline \multicolumn{5}{|l|}{ FABACEAE - FABOIDEAE } \\
\hline Amburana cearensis (Allemão) A.C. Sm. & Cumaru & & $\mathrm{X}$ & \\
\hline Erythrina velutina Willd. & Mulungú & $\mathrm{X}$ & $\mathrm{X}$ & $\mathrm{X}$ \\
\hline \multicolumn{5}{|l|}{ FABACEAE - MIMOSOIDEAE } \\
\hline Anadenanthera colubrina (Vell.) Brenan & Angico & & $\mathrm{X}$ & $\mathrm{X}$ \\
\hline Calliandra spinosa Ducke & Marizeira & $\mathrm{X}$ & $\mathrm{X}$ & $\mathrm{X}$ \\
\hline Chloroleucon sp. & Arapiraca & & $\mathrm{X}$ & \\
\hline Chloroleucon sp. & Saia-velha & & $\mathrm{X}$ & $\mathrm{X}$ \\
\hline Mimosa caesalpiniifolia Benth. & Sabiá & & $\mathrm{X}$ & $\mathrm{X}$ \\
\hline
\end{tabular}

Continua... 
TABELA 1: Continuação...

TABLE 1: Continued...

\begin{tabular}{|c|c|c|c|c|}
\hline \multirow{2}{*}{ Família/Espécie } & \multirow{2}{*}{ Nome vulgar } & \multicolumn{3}{|c|}{ Ambientes } \\
\hline & & I & II & III \\
\hline Mimosa tenuiflora (Wild.) Poir. & Jurema-preta & $\mathrm{X}$ & $\mathrm{X}$ & $\mathrm{X}$ \\
\hline Piptadenia sp. & Surucucu & & $\mathrm{X}$ & \\
\hline Piptadenia stipulacea (Benth.) Ducke & Jurema-branca & & $\mathrm{X}$ & $\mathrm{X}$ \\
\hline Senegalia sp. & Calumbí & & & $\mathrm{X}$ \\
\hline \multicolumn{5}{|l|}{ MALVACEAE } \\
\hline Guazuma ulmifolia Lam. & Mutamba & & $\mathrm{X}$ & $\mathrm{X}$ \\
\hline $\begin{array}{l}\text { Pseudobombax marginatum (A. St. -Hil., Juss. \& } \\
\text { Cambess.) A. Robyns }\end{array}$ & Embiratanha & & $\mathrm{X}$ & $\mathrm{X}$ \\
\hline \multicolumn{5}{|l|}{ OLACACEAE } \\
\hline Ximenia americana (Aubl.) Engl. & Ameixa & & & $\mathrm{X}$ \\
\hline \multicolumn{5}{|l|}{ POLYGONACEAE } \\
\hline Triplaris $s p$. & Cuaçú & & & $\mathrm{X}$ \\
\hline \multicolumn{5}{|l|}{ RHAMNACEAE } \\
\hline Ziziphus joazeiro Mart. & Juazeiro & & $\mathrm{X}$ & $\mathrm{X}$ \\
\hline \multicolumn{5}{|l|}{ INDETERMINADA } \\
\hline Indeterminada 3 & Pulga-de-leite & & $\mathrm{X}$ & $\mathrm{X}$ \\
\hline Indeterminada 4 & João-mole & & $\mathrm{X}$ & $\mathrm{X}$ \\
\hline
\end{tabular}

e Martins (2011), comunidades florestais em baixa densidade e riqueza de espécimes, seja ocasionados por fatores naturais e/ou antrópicos, proporciona uma maior distância entre o ponto central do quadrante e o indivíduo mais próximo, quando se realiza a amostragem pelo método dos Quadrantes, em estudos fitossociológicos.

Os efeitos da invasão por Cryptostegia madagascariensis se apresentam ainda mais evidentes, quando se analisou a estrutura das áreas. Dos 1.889 indivíduos ha ${ }^{-1}$ amostrados no Ambiente I, 1.557 pertencem à espécie invasora Cryptostegia madagascariensis, totalizando $82 \%$ da densidade total no referido ambiente. No entanto, nos Ambiente II e III a presença da espécie Cryptostegia madagascariensis foi bastante reduzida, totalizando apenas $14,2 \%$ e $9,6 \%$ da densidade, respectivamente, sendo estes valores inferiores aos encontrados em várias espécies nativas, como por exemplo Croton blanchetianus que obteve 26\% da densidade para o Ambiente II e 33,5\% para o Ambiente III. Com relação ao parâmetro frequência, Cryptostegia madagascariensis foi a única espécie observada em todos os pontos computados do Ambiente I, abrangendo $62 \%$ da frequência. Nos Ambientes II e III, Cryptostegia madagascariensis foi a segunda e terceira espécie com os maiores valores de frequência, 12,6\% e 10,1\% respectivamente (Tabela 2).

Para outros parâmetros analisados na vegetação, a agressividade da espécie invasora também foi evidenciada, tendo a área basal apresentado para o Ambiente I, um valor de aproximadamente 4,97 $\mathrm{m}^{2}$. ha ${ }^{-1}$, com destaque para as espécies Cryptostegia madagascariensis com $2,76 \mathrm{~m}^{2} \cdot \mathrm{ha}^{-1}$ e Copernicia prunifera

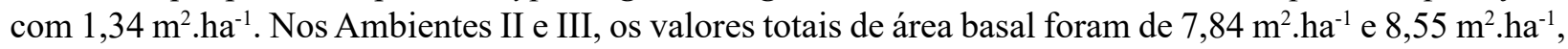
respectivamente. No Ambiente II, oito espécies apresentaram valores superiores à invasora, foram elas: Auxemma oncocalyx, Copernicia prunifera, Erythrina velutina, Croton blanchetianus, Calliandra spinosa, Mimosa caesalpiniifolia, Guazuma ulmifolia e Aspidosperma pyrifolium. No Ambiente III, onze espécies apresentaram valores maiores em relação à referida invasora, são elas: Copernicia prunifera, Auxemma oncocalyx, Mimosa tenuiflora, Croton blanchetianus, Guazuma ulmifolia, Poincianella pyramidalis, Erythrina velutina, Anadenanthera colubrina, Mimosa caesalpiniifolia, Pseudobombax marginatum 
e Jatropha molíssima. No entanto, nenhuma destas apresentou área basal maior que a da Cryptostegia madagascariensis no Ambiente I (Tabela 2).

TABELA 2: Parâmetros estruturais das comunidades estudadas no município de Ibaretama, Ceará, Brasil. Sendo: Ambiente I - forte intensidade de invasão; Ambiente II - Área de transição entre os ambientes I e III (média intensidade); Ambiente III - baixa intensidade de invasão.

TABLE 2: Structural parameters of the studied communities in Ibaretama, Ceará state, Brazil. Being: Ambience I - high intensity of invasion; Ambience II - Transition area between ambiences I and III (medium intensity); Ambience III - low intensity of invasion.

\begin{tabular}{|c|c|c|c|c|c|c|c|c|c|c|}
\hline \multicolumn{11}{|c|}{ Ambiente I } \\
\hline Nome Científico & $\mathrm{N}$ & $\mathrm{AB}$ & DA & DR & FA & FR & DoA & DoR & $\mathrm{VC}(\%)$ & VI $(\%)$ \\
\hline Cryptostegia madagascariensis & 428 & 2,7618 & 1557 & 82.31 & 100 & 62.50 & 13.119 & 55.59 & 68.80 & 66.70 \\
\hline Copernicia prunifera & 31 & 1,3404 & 99 & 5.96 & 17 & 10.63 & 6.367 & 26.98 & 16.12 & 14.29 \\
\hline Mimosa tenuiflora & 32 & 0,2517 & 104 & 6,15 & 17 & 10,63 & 1,195 & 5,070 & 5,28 & 7,06 \\
\hline Erythrina velutina & 3 & 0.3266 & 14 & 0.75 & 3 & 1.88 & 1.551 & 6.57 & 3.66 & 3.07 \\
\hline Parkinsonia aculeata & 9 & 0.0440 & 42 & 2.25 & 7 & 4.38 & 0.209 & 0.89 & 1.57 & 2.50 \\
\hline Jatropha molíssima & 8 & 0,0103 & 38 & 2,00 & 8 & 5,01 & 0,049 & 0,200 & 1,11 & 2,41 \\
\hline Calliandra spinosa & 3 & 0,2106 & 14 & 0.75 & 3 & 1.88 & 1.000 & 4.24 & 2.49 & 2.29 \\
\hline Combretum leprosum & 2 & 0,0041 & 9 & 0.50 & 1 & 0.63 & 0.020 & 0.08 & 0.29 & 0.40 \\
\hline Croton blanchetianus & 1 & 0,0133 & 4 & 0.25 & 1 & 0.63 & 0.063 & 0.27 & 0.26 & 0.38 \\
\hline Poincianella pyramidalis & 1 & 0,0023 & 4 & 0.25 & 1 & 0.63 & 0.011 & 0.05 & 0.15 & 0.31 \\
\hline Croton campestres & 1 & 0,0015 & 4 & 0.25 & 1 & 0.63 & 0.007 & 0.03 & 0.14 & 0.30 \\
\hline Cereus jamacaru & 1 & 0,0013 & 4 & 0.25 & 1 & 0.63 & 0.006 & 0.03 & 0.14 & 0.30 \\
\hline \multicolumn{11}{|c|}{ Ambiente II } \\
\hline Croton blanchetianus & 136 & 0,6362 & 2869 & 26.15 & 51 & 19.90 & 11.70 & 8.11 & 17.06 & 18.00 \\
\hline Cryptostegia madagascariensis & 65 & 0,3820 & 1563 & 12.50 & 32 & 12.66 & 7.027 & 4.87 & 9.52 & 10.57 \\
\hline Auxemma oncocalyx & 15 & 1,0907 & 643 & 2.88 & 16 & 6.20 & 20.06 & 13.91 & 9.87 & 8.65 \\
\hline Mimosa caesalpiniifolia & 29 & 0,4456 & 901 & 5.58 & 20 & 7.75 & 8.196 & 5.68 & 6.92 & 7.20 \\
\hline Copernicia prunifera & 27 & 0,9812 & 349 & 5.19 & 11 & 4.39 & 18.05 & 12.51 & 7.84 & 6.69 \\
\hline Mimosa tenuiflora & 38 & 0,3113 & 698 & 7.31 & 18 & 6.98 & 5.726 & 3.97 & 5.15 & 5.76 \\
\hline Erythrina velutina & 7 & 0,9064 & 128 & 1.17 & 4 & 1.55 & 16.67 & 11.56 & 6.36 & 4.76 \\
\hline Poincianella pyramidalis & 26 & 0,3050 & 478 & 4.33 & 14 & 5.43 & 5.610 & 3.89 & 4.11 & 4.55 \\
\hline Aspidosperma pyrifolium & 21 & 0,3911 & 386 & 3.50 & 12 & 4.65 & 7.194 & 4.99 & 4.24 & 4.38 \\
\hline Guazuma ulmifolia & 16 & 0,4434 & 294 & 2.67 & 8 & 3.10 & 8.155 & 5.65 & 4.16 & 3.81 \\
\hline Combretum leprosum & 22 & 0,2512 & 404 & 3.67 & 10 & 3.88 & 4.621 & 3.20 & 3.44 & 3.58 \\
\hline Jatropha molíssima & 39 & 0,0712 & 496 & 4.50 & 10 & 3.88 & 1.309 & 0.91 & 2.70 & 3.09 \\
\hline Calliandra spinosa & 5 & 0,5732 & 91 & 0.83 & 2 & 1.03 & 10.54 & 7.31 & 4.07 & 3.06 \\
\hline Cereus jamacaru & 16 & 0,0884 & 294 & 2.67 & 10 & 3.88 & 1.625 & 1.13 & 1.90 & 2.56 \\
\hline Ziziphus joazeiro & 5 & 0,2987 & 91 & 0.83 & 3 & 1.29 & 5.494 & 3.81 & 2.32 & 1.98 \\
\hline Indeterminada 3 & 16 & 0,0498 & 294 & 2.67 & 6 & 2.58 & 0.915 & 0.63 & 1.65 & 1.96 \\
\hline Piptadenia stipulacea & 10 & 0,0316 & 183 & 1.67 & 4 & 1.81 & 0.581 & 0.40 & 1.03 & 1.29 \\
\hline Indeterminada 2 & 7 & 0,0354 & 128 & 1.17 & 3 & 1.29 & 0.651 & 0.45 & 0.81 & 0.97 \\
\hline Chloroleucon sp. & 3 & 0,0850 & 55 & 0.50 & 2 & 0.78 & 1.564 & 1.08 & 0.79 & 0.79 \\
\hline Pseudobombax marginatum & 2 & 0,2327 & 36 & 0,34 & 1 & 0,52 & 4,279 & 2,97 & 1,65 & 1,27 \\
\hline Amburana cearensis & 2 & 0,0387 & 36 & 0.33 & 1 & 0.52 & 0.712 & 0.49 & 0.41 & 0.45 \\
\hline Libidibia ferrea & 2 & 0,0293 & 36 & 0.33 & 1 & 0.52 & 0.540 & 0.37 & 0.35 & 0.41 \\
\hline Indeterminada 4 & 2 & 0,0243 & 36 & 0.33 & 1 & 0.52 & 0.446 & 0.31 & 0.32 & 0.39 \\
\hline Crateva tapia & 1 & 0,0491 & 18 & 0.17 & 0.6 & 0.26 & 0.903 & 0.63 & 0.40 & 0.35 \\
\hline Chloroleucon sp. & 2 & 0,0075 & 36 & 0.33 & 1 & 0.52 & 0.139 & 0.10 & 0.21 & 0.32 \\
\hline Anadenanthera colubrina & 2 & 0,0020 & 36 & 0.33 & 1 & 0.52 & 0.036 & 0.03 & 0.18 & 0.29 \\
\hline
\end{tabular}


TABELA 2: Continuação...

TABLE 2: Continued...

\begin{tabular}{|c|c|c|c|c|c|c|c|c|c|c|}
\hline \multicolumn{11}{|c|}{ Ambiente I } \\
\hline Nome Científico & $\mathrm{N}$ & $\mathrm{AB}$ & DA & DR & FA & FR & DoA & DoR & $\mathrm{VC}(\%)$ & VI $(\%)$ \\
\hline Myracrodruon urundeuva & 1 & 0,0117 & 18 & 0.17 & 0.6 & 0.26 & 0.215 & 0.15 & 0.16 & 0.19 \\
\hline Piptadenia sp. & 1 & 0,0038 & 18 & 0.17 & 0.6 & 0.26 & 0.071 & 0.05 & 0.11 & 0.16 \\
\hline Croton campestres & 1 & 0,0030 & 18 & 0.17 & 0.6 & 0.26 & 0.056 & 0.04 & 0.10 & 0.15 \\
\hline Bauhinia cheilantha & 1 & 0,0030 & 18 & 0.17 & 0.6 & 0.26 & 0.056 & 0.04 & 0.10 & 0.15 \\
\hline \multicolumn{11}{|c|}{ Ambiente III } \\
\hline Croton blanchetianus & 174 & 0,7106 & 1197 & 33.46 & 63 & 23.31 & 4.891 & 8.30 & 20.88 & 21.69 \\
\hline Copernicia prunifera & 31 & 1,8873 & 213 & 5.96 & 19 & 7.02 & 12.99 & 22.06 & 14.01 & 11.68 \\
\hline Poincianella pyramidalis & 47 & 0,6755 & 323 & 9.04 & 30 & 11.24 & 4.649 & 7.89 & 8.47 & 9.39 \\
\hline Auxemma oncocalyx & 23 & 1,4995 & 158 & 4.42 & 16 & 5.90 & 10.32 & 17.52 & 10.97 & 9.28 \\
\hline Mimosa tenuiflora & 45 & 0,8408 & 309 & 8.65 & 23 & 8.71 & 5.787 & 9.83 & 9.24 & 9.06 \\
\hline Cryptostegia madagascariensis & 50 & 0,1650 & 344 & 9.62 & 27 & 10.11 & 1.136 & 1.93 & 5.77 & 7.22 \\
\hline Jatropha molissima & 32 & 0,1793 & 220 & 6.15 & 16 & 6.18 & 1.234 & 2.09 & 4.12 & 4.81 \\
\hline Guazuma ulmifolia & 10 & 0,7781 & 68 & 1.92 & 7 & 2.81 & 5.356 & 9.09 & 5.51 & 4.61 \\
\hline Combretum leprosum & 14 & 0,1122 & 96 & 2.69 & 10 & 3.93 & 0.772 & 1.31 & 2.00 & 2.65 \\
\hline Erythrina velutina & 4 & 0,5047 & 27 & 0.77 & 3 & 1.12 & 3.474 & 5.90 & 3.33 & 2.60 \\
\hline Mimosa caesalpiniifolia & 13 & 0,2165 & 89 & 2.50 & 5 & 1.97 & 1.491 & 2.53 & 2.52 & 2.33 \\
\hline Anadenanthera colubrina & 8 & 0,2419 & 55 & 1.54 & 6 & 2.25 & 1.665 & 2.83 & 2.18 & 2.20 \\
\hline Cereus jamacaru & 9 & 0,0507 & 61 & 1.73 & 6 & 2.53 & 0.349 & 0.59 & 1.16 & 1.62 \\
\hline Pseudobombax marginatum & 5 & 0,2016 & 34 & 0.96 & 3 & 1.12 & 1.388 & 2.36 & 1.66 & 1.48 \\
\hline Senegalia sp. & 10 & 0,0367 & 68 & 1.92 & 5 & 1.97 & 0.252 & 0.43 & 1.18 & 1.44 \\
\hline Piptadenia stipulacea & 6 & 0,0355 & 41 & 1.15 & 3 & 1.40 & 0.244 & 0.41 & 0.78 & 0.99 \\
\hline Chloroleucon sp.2 & 2 & 0,1317 & 13 & 0.38 & 1 & 0.56 & 0.907 & 1.54 & 0.96 & 0.83 \\
\hline Spondias mombin & 2 & 0,1145 & 13 & 0.38 & 1 & 0.56 & 0.788 & 1.34 & 0.86 & 0.76 \\
\hline Ziziphus joazeiro & 4 & 0,0146 & 27 & 0.77 & 3 & 1.12 & 0.101 & 0.17 & 0.47 & 0.69 \\
\hline Ximenia americana & 5 & 0,0198 & 34 & 0.96 & 2 & 0.84 & 0.136 & 0.23 & 0.60 & 0.68 \\
\hline Indeterminada 3 & 4 & 0,0197 & 27 & 0.77 & 2 & 0.84 & 0.136 & 0.23 & 0.50 & 0.61 \\
\hline Croton campestris & 3 & 0,0036 & 20 & 0.58 & 2 & 0.84 & 0.025 & 0.04 & 0.31 & 0.49 \\
\hline Commiphora leptophloeos & 4 & 0,0224 & 27 & 0.77 & 0.7 & 0.28 & 0.154 & 0.26 & 0.52 & 0.44 \\
\hline Bauhinia cheilantha & 3 & 0,0035 & 20 & 0.58 & 1.5 & 0.56 & 0.024 & 0.04 & 0.31 & 0.39 \\
\hline Triplaris $s p$ & 1 & 0,0491 & 6 & 0.19 & 0.7 & 0.28 & 0.338 & 0.57 & 0.38 & 0.35 \\
\hline Aspidosperma pyrifolium & 2 & 0,0022 & 13 & 0.38 & 1.5 & 0.56 & 0.015 & 0.03 & 0.21 & 0.32 \\
\hline Calliandra spinosa & 2 & 0,0020 & 13 & 0.38 & 1.5 & 0.56 & 0.014 & 0.02 & 0.20 & 0.32 \\
\hline Croton sp. & 2 & 0,0164 & 13 & 0.38 & 0.7 & 0.28 & 0.113 & 0.19 & 0.29 & 0.29 \\
\hline Libidibia ferrea & 1 & 0,0133 & 6 & 0.19 & 0.7 & 0.28 & 0.091 & 0.16 & 0.17 & 0.21 \\
\hline Myracrodruon urundeuva & 1 & 0,0016 & 6 & 0.19 & 0.7 & 0.28 & 0.011 & 0.02 & 0.11 & 0.16 \\
\hline Indeterminada 4 & 1 & 0,0011 & 6 & 0.19 & 0.7 & 0.28 & 0.007 & 0.01 & 0.10 & 0.16 \\
\hline $\begin{array}{r}\text { Total }-\mathrm{I} \\
\end{array}$ & 520 & 4,9680 & 1889 & 100 & 160 & 100 & 23.598 & 100 & 100 & 100 \\
\hline Total - II & 520 & 7,8429 & 10651 & 100 & 258 & 100 & 144.25 & 100 & 100 & 100 \\
\hline Total - III & 520 & 8,5569 & 3579 & 100 & 273 & 100 & 58.898 & 100 & 100 & 100 \\
\hline
\end{tabular}

Em que: N - Números de Indivíduos; AB - Área Basal; DA - Densidade Absoluta; DR - Densidade Relativa; FA -

Frequência Absoluta; FR - Frequência Relativa; DoA - Dominância Absoluta; DoR - Dominância Relativa; VC (\%)

- Valor de Cobertura; VI (\%) - Valor de Importância.

Where: N - Number of Individuals; AB - Basal Area; DA - Absolute Density; DR - Relative Density; FA - Absolute

Frequency; FR - Relative Frequency; DoA - Absolute Dominance; DoR - Relative Dominance; VC (\%) - Coverage

Value; VI (\%) - Importance Value. 
Segundo Pegado et al. (2006) e Souza et al. (2011), uma das principais características das plantas invasoras na caatinga é formar maciços populacionais em áreas nas quais se estabelecem. Isso foi constatado na área com forte invasão por Cryptostegia madagascariensis (Ambiente I). Conforme Andrade (2013) esta espécie forma grandes maciços populacionais o que, consequentemente, dificulta o estabelecimento e a sobrevivência de espécies autóctones. Desta forma, o referido táxon aumentou sua capacidade de produção de biomassa, refletindo em maiores abundância e dominância em comparação aos nativos da região, como pode ser observado na Figura 2.

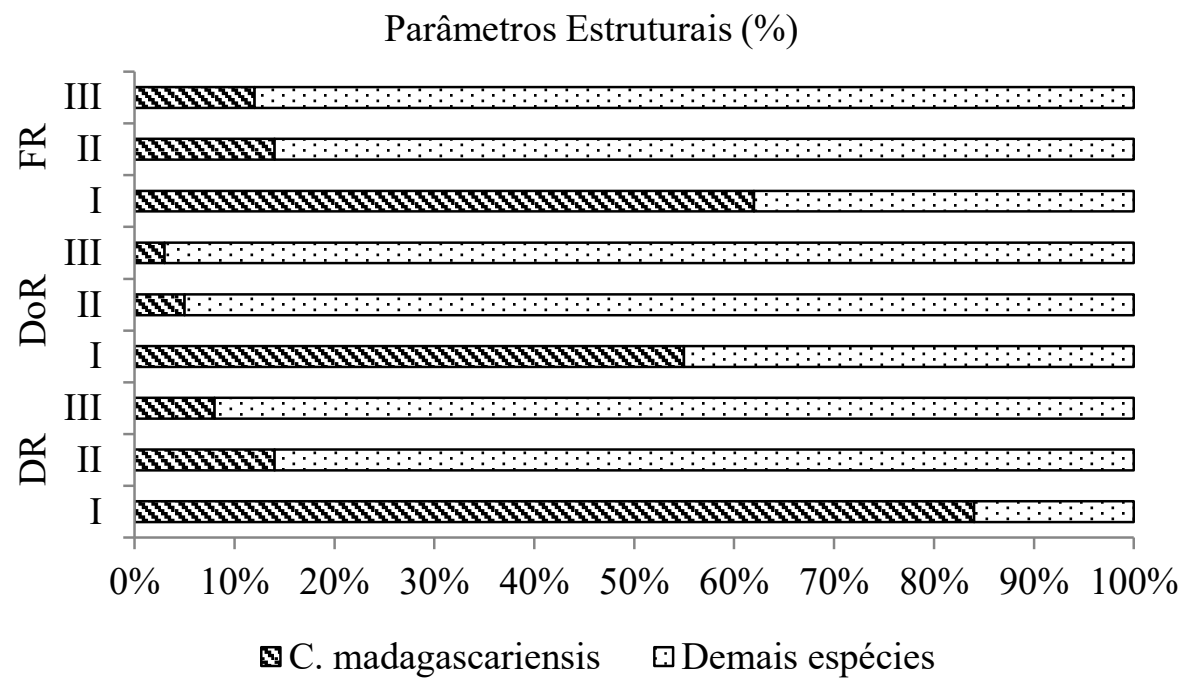

FIGURA 2: Parâmetros estruturais da Cryptostegia madagascariensis e das demais espécies nos ambientes avaliados. Sendo: $\mathrm{I}$ = forte intensidade de invasão; II = Área de Transição entre os Ambientes I e III (média intensidade); III = baixa intensidade de invasão; DR - Densidade Relativa; DoR - Dominância Relativa; FR - Frequência Relativa.

FIGURE 2: Structural parameters of Cryptostegia madagascariensis and other species in the evaluated environments. Being: I - high intensity of invasion; II - Transition Areas between Ambiences I and III (medium intensity); III - low intensity of invasion; RD - Relative Density; RDo Relative Dominance; RF - Relative Frequency.

Com relação ao Valor de Importância (VI) no Ambiente I, observa-se que Cryptostegia madagascariensis foi responsável por $66,7 \%$ do valor total, enquanto que no Ambiente II, a espécie de maior valor de importância foi Croton blanchetianus com 18\%, seguido da invasora com 10,6\%. No Ambiente III, nota-se que a importância da espécie invasora continuou a diminuir, representando apenas $7,2 \%$. Neste ambiente as espécies nativas Croton blanchetianus, Copernicia prunifera, Poincianella pyramidalis, Auxemma oncocalyx e Mimosa tenuiflora apresentaram valores de importância superiores à referida invasora, mostrando uma melhor distribuição, o que certamente advém de um estágio sucessional mais avançado e de um ecossistema mais equilibrado (Tabela 2).

Os dados supracitados mostram a capacidade que Cryptostegia madagascariensis tem de dominar as áreas nas quais se estabelecem. Pegado et al. (2006) e Andrade, Fabricante e Oliveira (2010), avaliaram áreas de caatinga invadidas por Prosopis juliflora na Paraíba e também encontraram altos valores de importância para esta espécie, com 70,8\% e 92\%, respectivamente. Noutro trabalho desenvolvido por Souza (2012) na Paraíba, a espécie Sesbania virgata apresentou altos valores de VI, alcançando 78\%. Segundo Andrade, Fabricante e Oliveira (2009), os altos valores de VI observados em estudos na Caatinga invadida por espécies invasoras revelam a agressividade com que dominam o ambiente e os impacto que elas causam sobre a biodiversidade local.

Os valores de diversidade pelo Índice de Shannon-Weiner $\left(H^{\prime}\right)$ e Índice de Pielou $\left(J^{\prime}\right)$ foram baixos 
para o Ambiente I ( 0,85 e 0,32, respectivamente) e maiores para as demais áreas (Amb. $2=2,67$ e 0,76; Amb. $3=2,50$ e 0,72). O Índice de Impacto Ambiental de Exóticas (IIAE) calculados foram -0,33, 0,78 e 0,84 , para os ambientes I, II e III, respectivamente (Tabela 3 ).

TABELA 3: Valores de diversidade de Shannon-Wiener $\left(H^{\prime}\right)$, de equabilidade de Pielou $\left(J^{\prime}\right)$ e do Índice de Impacto Ambiental de Exóticas (IIAE).

TABLE 3: Values of diversity of Shannon-Wiener $\left(H^{\prime}\right)$, Pielou equability $\left(J^{\prime}\right)$ and the Index of Environmental Impact of Exotics (IEIE).

\begin{tabular}{cccc}
\hline \multirow{2}{*}{ Índices } & \multicolumn{3}{c}{ Ambientes } \\
\cline { 2 - 4 } & I & II & III \\
\hline H' & 0,85 & 2,67 & 2,50 \\
J' & 0,32 & 0,76 & 0,72 \\
IIAE & $-0,33$ & 0,78 & 0,84 \\
\hline
\end{tabular}

Os valores de IIAE evidenciados acima indicam novamente que a área com forte intensidade de invasão encontra-se fortemente impactada pela ocupação da invasora Cryptostegia madagascariensis, pois, o índice do IIAE varia de -1 a 1 e quando o valor analisado é -1, implica que a área não possui plantas nativas e quando o valor analisado é 1, a área não possui plantas exóticas (REASER et al., 2007). Portanto, os valores negativos para o Ambiente I indicam que a invasora causou alterações na florística e na estrutura da comunidade estudada. Segundo Andrade, Fabricante e Oliveira (2010), valores negativos já representam sérios problemas ambientais para as comunidades autóctones.

Com relação ao índice de diversidade dos Ambiente II e III, observou-se que o valor foi semelhante ao de vários outros trabalhos desenvolvidos em caatinga preservadas (PEGADO et al., 2006; SANTANA; SOUTO, 2006; PESSOA et al., 2008; ANDRADE; FABRICANTE; OLIVEIRA, 2009; 2010; ALMEIDA NETO et al., 2009; FABRICANTE; ANDRADE; TERCEIRO, 2012). Porém, o índice do Ambiente I demonstrou estar bastante baixo, quando comparado aos índices de $H^{\prime}$ das áreas adjacentes do presente estudo, bem como aos índices dos trabalhos supracitados, o que é comum ocorrer em áreas impactadas por espécies exóticas invasoras (PEGADO et al., 2006; ANDRADE; FABRICANTE; OLIVEIRA, 2009; 2010; SOUZA et al., 2011; SOUZA, 2012).

Rascher et al. (2011) também constataram baixos índices de diversidade para áreas invadidas por Acacia longifolia nas florestas nativas de Portugal, observando diminuição de mais de $50 \%$ quando comparadas áreas invadidas com as não invadidas. Vilà et al. (2011), em uma meta-análise global de 199 artigos sobre os impactos das invasões em comunidades e ecossistemas, observaram que um dos resultados mais notáveis em estudos de invasão é a redução da diversidade de espécies de plantas autóctones devido ao aumento de populações invasoras. Andrade, Fabricante e Oliveira (2010), corroboram que a baixa diversidade e equabilidade observadas em comunidades invadidas são comuns, em consequência da alta abundância de indivíduos da espécie invasora.

Logo, pode-se afirmar que a participação da Cryptostegia madagascariensis alterou a fitodiversidade da área fortemente invadida quanto à composição florística e à estrutura da comunidade estudada, tornando-a distinta em relação às demais áreas e reduzindo a importância das espécies nativas nas comunidades afetadas.

Destaque-se ainda que, além de todos os danos estruturais causados nas comunidades invadidas, as invasões biológicas tendem a alterar as propriedades ecológicas essenciais como ciclo de nutrientes, produtividade, estrutura da comunidade, distribuição de biomassa, acúmulo de serapilheira e relações entre plantas e polinizadores (LOPES, 2009; LEÃO et al., 2011). Assim, pode-se afirmar que na área invadida por Cryptostegia madagascariensis outros componentes do ecossistema podem estar alterados além da composição e estrutura vegetal.

Além disso, a ação antrópica evidenciada na área fortemente invadida - corte raso da vegetação 
- contribuiu para a expansão desta invasora, aumentando sua área total ocupada, sua abundância local e capacidade de dispersão. Segundo Ziller e Zalba (2007), quanto maior o grau de perturbação de um ecossistema natural, maior o potencial de dispersão e estabelecimento de espécies exóticas, especialmente após a redução da diversidade natural pela exploração excessiva. Para Martins (2012), as ações de cunho antrópico são agentes de perturbação que podem modificar a funcionalidade e a composição florística de uma comunidade, deixando-a vulnerável a vários outros problemas que podem agravar as modificações estruturais da vegetação, sendo a invasão biológica uma delas.

\section{CONCLUSÕES}

A invasão biológica por Cryptostegia madagascariensis reduz a riqueza de espécies e altera fortemente a estrutura das comunidades vegetais da Caatinga.

Áreas mais jovens submetidas a um grau de antropismo elevado dificultam a autorregeneração das espécies nativas, favorecendo o estabelecimento e a dispersão da invasora, levando as áreas invadidas a uma condição ecologicamente inferior, com perda de biodiversidade no nível de espécies e de processos ecológicos.

Os resultados chamam a atenção para implementação de ações voltadas para controle, manejo e recuperação de ambientes invadidos por esta espécie no bioma Caatinga.

\section{AGRADECIMENTO}

Ao Projeto Biomas/EMBRAPA/CNA, pelos recursos financeiros indispensáveis para realização deste trabalho, e a toda equipe do Laboratório de Ecologia Vegetal da Universidade Federal da Paraíba, Campus de Areia - PB.

\section{REFERÊNCIAS}

ALMEIDA NETO, J. X. et al. Composição florística, estrutura e análise populacional do Feijão-bravo (Capparis flexuosa L.) no semiárido Paraibano, Brasil. Caatinga, Mossoró, v. 22, n. 4, p. 187-194, 2009.

ANDRADE, L. A. Plantas invasoras: espécies exóticas invasoras da caatinga e ecossistemas associados. 1. ed. Campina Grande: Epgraf, 2013. 100 p.

ANDRADE, L. A.; FABRICANTE, J. R.; OLIVEIRA, F. X. Impactos da invasão de Prosopis juliflora (Sw.) DC. (Fabaceae) sobre o estrato arbustivo-arbóreo em áreas de Caatinga no Estado da Paraíba, Brasil. Acta Scientiarum, Biological Sciences, Maringá, v. 32, n. 3, p. 249-255, 2010.

ANDRADE, L. A.; FABRICANTE, J. R.; OLIVEIRA, F. X. Invasão biológica por Prosopis juliflora (Sw.) DC.: impactos sobre a diversidade e a estrutura do componente arbustivo-arbóreo da caatinga no Estado do Rio Grande do Norte, Brasil. Acta Botanica Brasilica, São Paulo, v. 23, n. 4, 2009.

ANGIOSPERM PHYLOGENY GROUP III. An update of the Angiosperm Phylogeny Group classification for the orders and families of flowering plants: APG III. Botanical Journal of the Linnean Society, Malden, v. 161, p. 105-121, 2009.

BRUMMITT, R. K., POWELL, C. E. Authors of plant names. Kew: Royal Botanic Gardens, 1992.732 p. COTTAM, G.; CURTIS, J. T. The use of distance measures in phytosociological sampling. Ecology, Washington, v. 37, n. 3, p. 451-460, 1956.

ENDRESS, M. E.; BRUYNS, P. V. A revised classification of the Apocynaceae s.l. The Botanical Review, New York, v. 66, p. 1-56, 2000.

FABRICANTE, J. R.; ANDRADE, L. A.; TERCEIRO, R. G. D. Divergências na composição e na estrutura do componente arbustivo-arbóreo entre duas áreas de caatinga na região do Submédio São Francisco (Petrolina, PE/Juazeiro, BA). Biotemas, Florianópolis, v. 25, n. 3, p. 97-109, 2012.

FUNDAÇÃO CEARENSE DE METEOROLOGIA E RECURSOS HÍDRICOS; INSTITUTO DE PESQUISA E ESTRATÉGIA ECONÔMICA DO CEARÁ. Perfil básico municipal, Ibaretama, 2013. Disponível em: $<$ http://www.ipece.ce.gov.br/publicacoes/perfil_basico/pbm-2013/Ibaretama.pdf $>$. Acesso em: 13 jan. 2014. 
KLACKENBERG, J. Revision of the genus Cryptostegia R. Br. (Apocynaceae, Periplocoideae). Adansonia, Paris, v. 23, n. 2, p. 205-218, 2001.

KRITICOS, D. J. et al. Climate change and biotic invasions: a case history of a tropical woody vine. Biological Invasions, Knoxville, v. 5, p. 145-165, 2003.

LEÃO, T. C. C. et al. Espécies exóticas invasoras no Nordeste do Brasil: contextualização, manejo e políticas públicas. Recife: Centro de Pesquisas Ambientais do Nordeste; Instituto Hórus de Desenvolvimento e Conservação Ambiental, 2011. 99 p.

LOPES, R. M. Informe sobre as espécies exóticas invasoras marinhas no Brasil. Brasília: MMA; SBF, 2009. 440 p. (Série Biodiversidade, 33).

MARANGON, L. C. et al. Estrutura fitossociológica e classificação sucessional do componente arbóreo de um fragmento de floresta estacional semidecidual, no município de Viçosa, Minas Gerais. Cerne, Lavras, v. 13, n. 2, p. 208-221, 2007.

MARTINS, S. V. Ecologia de Florestas Tropicais do Brasil. 2. ed. Viçosa, MG: Editora UFV, 2012. p. 371.

MATEUCCI, S. D.; COLMA, A. Metodologia para el estudio de la vegetacion. Washington: Programa Regional de Desarrolo Científico y Tecnológico, 1982. 168 p.

MORO, M. F.; MARTINS, F. R. Métodos de levantamento do componente arbóreo-arbustivo. In: FELFILI, J. M. et al. Fitossociologia no Brasil: métodos e estudos de casos. 1. ed. Viçosa, MG: Editora UFV, 2011. p. 556.

MUELLER-DOMBOIS, D.; ELLENBERG, H. Aims and methods of vegetation Ecology. New York: John Wiley \& Sons, 1974. 547 p.

ODUM, E. P. Ecologia. Rio de Janeiro: Guanabara, 1988. 434 p.

OLIVEIRA, A. E. S.; PEREIRA, D. G. Erradicação de espécies exóticas invasoras: múltiplas visões da realidade brasileira. Desenvolvimento e Meio Ambiente, Curitiba, n. 21, p. 173-181, 2010.

OLIVEIRA, M. C.; SILVA JÚNIOR, M. C.; RIBEIRO, J. F. Perturbações e invasões biológicas: ameaças para a Biodiversidade nativa? Revista CEPPG, Catalão, n. 25, v. 2, p. 166-183, 2011.

PARQUE DE CIÊNCIA E TECNOLOGIA (SP). Mata Nativa: sistema para análise fitossociológica e elaboração de planos de manejo de florestas nativas. São Paulo: CIENTEC, 2002. 126 p.

PEGADO, C. M. A. et al. Efeito da invasão biológica de Prosopis juliflora (Sw.) DC. Sobre a composição e a estrutura do estrato arbustivo-arbóreo da caatinga no Município de Monteiro - PB, Brasil. Acta Botânica Brasilica, São Paulo, v. 20, p. 889-898, 2006.

PESSOA, M. F. et al. Estudo da cobertura vegetal em ambientes da caatinga com diferentes formas de manejo no assentamento Moacir Lucena, Apodi - RN. Caatinga, Mossoró, v. 21, n. 3, p. 40-48, 2008.

RASCHER, K. G. et al. Acacia longifolia invasion impacts vegetation structure and regeneration dynamics in open dunes and pine forests. Biological Invasions, Knoxville, v. 13, p. 1099-1113, 2011.

REASER, J. K. et al. Ecological and socioeconomic impacts of invasive alien species in island ecosystems. Environmental Conservation, Berna, v. 34, n. 2, p. 98-111, 2007.

RODAL, M. J. N. F.; SAMPAIO, E. V. S. B.; FIGUEIREDO, M. A. Manual sobre métodos de estudos florísticos e fitossociológicos: ecossistema caatinga. Brasília: Sociedade Botânica do Brasil, 2013.

SANTANA, J. A S.; SOUTO, J. S. Diversidade e Estrutura Fitossociológica da Caatinga na Estação Ecológica do Seridó-RN. Biologia e Ciência da Terra, São Cristóvão, v. 6, n. 2, p. 232-242, 2006.

SHANNON, C. E.; WEAVER W. The mathematical theory of communication. Urbana: University of Illinois Press, 1949.

SILVA, C. M. et al. Guia de plantas: visitadas por abelhas na Caatinga. Fortaleza: Editora Fundação Brasil Cidadão, 2012.99 p.

SOUZA, V. C. et al. Avaliação populacional de Sesbania virgata (Cav.) Pers. (Fabaceae Lindl.), nas margens do rio Paraíba. Revista Brasileira de Ciências Agrárias, Recife, v. 6, n. 2, p. 314-320, 2011.

SOUZA, V. C. Invasão biológica por Sesbania virgata (CAV.) PERS. na Paraíba, Brasil: biologia reprodutiva, ecofisiologia de sementes e estrutura populacional. 2012. 199 f. Tese (Doutorado) - Programa de Pós-graduação em Agronomia, Universidade Federal da Paraíba, Areia, 2012.

VIEIRA, M. F. et al. Biologia reprodutiva de cryptostegia madagascariensis Bojer ex decne. (periplocoideae, apocynaceae), espécie ornamental e exótica no Brasil. Bragantia, Campinas, v. 63, n. 3, p. 325-334, 2004. 
VILÀ, M. et al. Ecological impacts of invasive alien plants: a meta-analysis of their effects on species, communities and ecosystems. Ecology Letters, Nova Jersey, v. 14, p. 702-708, 2011.

XAVIER, Klerton Rodrigues Forte. Análise Florística e Fitossociológica em dois Fragmentos de Floresta Serrana no Município de Dona Inês, Paraíba. 2009. 76 f. Dissertação (Mestrado) - Programa de Pósgraduação em Agronomia, Universidade Federal da Paraíba, Areia, 2009.

ZENNI, R. D.; ZILLER, S. R. An overview of invasive plants in Brazil. Revista Brasileira de Botânica, São Paulo, v. 34, n. 3, p. 431-446, 2011.

ZILLER, S. R.; ZALBA, S. Propostas de ação para prevenção e controle de espécies exóticas invasoras. Natureza e Conservação, Curitiba, v. 5, n. 2, p. 8-15, 2007. 